\title{
Acidentes de transporte terrestre em cidade da Região Sul do Brasil: avaliação da cobertura e qualidade dos dados
}

\author{
Traffic accidents in a city in Southern Brazil: \\ an evaluation of coverage and quality of data
}

Sel ma Maffei de Andrade 1

Maria Helena Prado de Mello-Jorge 2

\footnotetext{
1 Departamento Materno-Infantil e Saúde Comunitária, Universidade Estadual de Londrina. Rua Pernambuco 1227 204, Londrina, PR 86020-071, Brasil. semaffei@sercomtel.com.br 2 Departamento de Epidemiologia, Faculdade de Saúde Pública, Universidade de São Paulo. Av. Dr. Arnaldo 715, São Paulo, SP 01246-904, Brasil.
}

Abstract The aim of this study was to analyze police coverage and the validity of data on emergency and hospitalization records as well as on death certificates for traffic casualties in Londrina, Paraná State, Brazil. Victims $(3,643)$ of road accidents during the first semester of 1996 were investigated and followed up after 180 days to confirm whether death was due to the accident. Police data recorded only $32.5 \%$ of the casualties, the coverage being higher for car occupants (71.6\%) and lower for cyclists (8.1\%) and pedestrians (24.8\%). Agreement was low between original information and that derived from investigation of death certificates (Kappa coefficient 0.10; $95 \% \mathrm{Cl}: 0.02-0.17$ ), fair for hospitalization records (Kappa coefficient $0.33 ; 95 \% \mathrm{Cl}: 0.27$ 0.40), and substantial for emergency records (Kappa coefficient 0.63; 95\% Cl: 0.61-0.65). Results suggest that police data underestimate the number of traffic casualties and that it is necessary to improve the validity of medical records.

Key words Traffic Accidents; Hospital Records; Death Certificates

Resumo O objetivo do estudo foi o de avaliar a cobertura policial e a validade dos dados registrados em fichas de pronto-socorro, internação e nas declarações de óbito de víti mas de acidentes de transporte terrestre ocorridos em Londrina, Paraná, Brasi I. Foram estudadas 3.643 vítimas resultantes de aci dentes ocorridos no primei ro semestre de 1996, para as quais observou-se um prazo de seguimento de 180 dias para verificar a ocorrência de óbito devido ao acidente. A cobertura policial desses aci dentes foi bastante baixa (32,5\%) sendo maior para os ocupantes de carro $(71,6 \%)$ e menor para ciclistas $(8,1 \%)$ e pedestres $(24,8 \%)$. A concordância entre as informações regi stradas ori gi nalmente e a obti da após investi gação foi pequena nas declarações de óbi to (coefi ciente Kappa 0,10; IC 95\%: 0,02-0,17) regular nas fichas de internação (coeficiente Kappa 0,33; IC 95\%: 0,27-0,40) e substancial nas fichas de pronto-socorro (coefi ciente Kappa 0,63; IC 95\%: 0,61-0,65). Os resultados indicam que os registros pol iciais subestimam o número de vítimas por esses aci dentes. Sugerem, ainda, ser necessário investir na mel horia da quali dade dessas informações, principalmente nos níveis de internação e óbito.

Palavras-chave Acidentes deTrânsito; Registros Hospitalares; Atestados de Óbito 


\section{Introdução}

A importância da qualidade da informação sobre as circunstâncias dos acidentes e violências produtoras de lesões (causas externas de morbi-mortalidade) para o adequado planejamento de ações preventivas, já vem sendo destacada há vários anos, tanto no Brasil (Mello-Jorge, 1990; Minayo \& Souza, 1993) como em outros países (Lapidus et al., 1994; Moyer et al., 1989). Como enfatizam Lebrão et al. (1997:37), essa informação é importante, pois "não se previne a fratura, a quei madura ou o traumatismo crânio-encefálico mas, sim, a queda; o acidente com fogo ou o aci dente automobi lístico". Dessa forma, torna-se imprescindível avaliar, em nível local, a validade dessas informações nos diversos níveis de atenção à vítima traumatizada em acidentes de transporte terrestre, tipo predominante de causa externa de lesões e óbitos em várias cidades de médio porte, incluindo Londrina, no Estado do Paraná (Andrade, 1995). Tal análise pode contribuir para fornecer subsídios tanto para gestores locais das áreas de planejamento urbano e de saúde, como para o ensino de graduação no que se refere ao registro adequado da informação em saúde.

Com relação à cobertura dos eventos acidentais ou violentos, os estudos são mais escassos e dependem, em grande parte, de um adequado "padrão-ouro", isto é, o melhor padrão de referência para quantificar tais eventos, principalmente os não fatais. No caso da mortalidade, muitas declarações de óbito, não obstante informarem tratar-se de morte por causa não natural (externa), deixam de detaIhar o tipo específico de causa (acidente de transporte, queda, agressão, etc.) que provocou a lesão fatal, subestimando-as e levando a um aumento dos "eventos cuja intenção é indeterminada" (códigos Y10 a Y34 da Classificação Internacional de Doenças, décima revisão - CID-10 -, atualmente em vigor no Brasil) (OMS, 1996).

Os registros policiais propiciam, em geral, dados mais detal hados sobre as circunstâncias dos acidentes de transporte terrestre, como o tipo de veículo envolvido, condições climáticas e da pista no momento do acidente, entre outras. Possuem, ainda, a vantagem de captar vítimas não fatais, ao contrário do Sistema de Informações de Mortalidade (SIM) possibilitando análise de tendências não somente para os óbitos. Todavia, alguns estudos internacionais revelam que nem todas as vítimas traumatizadas nesses ti pos de acidente são registradas pela polícia (Aptel et al., 1999). Conhecer em que medida esses registros estão cobrindo tais aci- dentes pode contribuir para estimativas que possibilitem mel hor avaliar a magnitude desses eventos, tanto na produção de vítimas fatais, como de sobreviventes.

Os objetivos deste trabal ho foram, portanto, o de avaliar a cobertura dos registros policiais quanto à captação de vítimas de acidentes de transporte terrestre ocorridos em Londrina, bem como o de analisar a qualidade (validade) dos registros médicos com relação à informação sobre as circunstâncias do acidente, nas fichas de atendimento de pronto-socorro, internação e na declaração de óbito.

\section{Material e método}

Foram estudadas 3.643 vítimas de acidentes de transporte terrestre - rubricas V01 a V89 da CID-10 (OMS, 1996). Essas vítimas resultaram de acidentes ocorridos no período compreendido entre 1o de janeiro a 30 de junho de 1996, no Município de Londrina (área urbana e rural) que se localiza ao norte do Estado do Paraná, Região Sul, com uma população, em 1996, de 421.343 habitantes (IBGE, s.d.). Para cada uma dessas vítimas foi observado um prazo de 180 dias para verificar a ocorrência de óbito devido ao acidente, porque nesse prazo ocorre quase a totalidade dessas mortes (Mello-Jorge, 1980).

As fontes de dados foram múltiplas: Declarações de Óbito (DO), Boletins de Ocorrência (BOs) sobre os acidentes, elaborados pela Polícia Militar, fichas do Sistema Único de Saúde (SUS) de atendimento em prontos-socorros e de Autorização de Internação Hospitalar (AIH) de todos os hospitais gerais (seis) e ortopédico (um) da cidade. Todas as fichas (de atendimento a trauma ou clínico) foram revisadas manual mente e, nos casos de acidentes de transporte terrestre ou trauma de intenção indeterminada, transcritas para formulário próprio da pesquisa. Como fontes complementares de informação foram utilizadas a imprensa escrita, as Comunicações de Acidentes de Trabalho (CATs) e entrevistas com familiares ou vítimas, nos casos em que as fichas disponíveis não permitiam um detalhamento quanto às circunstâncias do acidente. Com base nesses levantamentos, foram criados, por meio do programa de domínio público Epi Info (CDC/WHO, 1996), quatro bancos de dados independentes (sobre casos de acidente, óbito, internação e atendimento em pronto-socorro), os quais foram cruzados por meio do nome das vítimas e data do acidente. Detalhes a respeito da metodologia estão descritos em outro trabalho (Andrade \& Mello-Jorge, 2000). 
A avaliação da qualidade dos registros médicos (em prontos-socorros, da ficha de internação ou da Declaração de Óbito) em relação às circunstâncias do acidente, tomou como base a informação inicial registrada nas respectivas fichas comparada à informação pós-investigação, que levou em consideração os detalhes proporcionados pelos BOs, CATs, notícias veiculadas na imprensa escrita e/ ou entrevistas com os familiares ou a própria vítima. Nessa avaliação, tomou-se como parâmetro os agrupamentos das rubricas da CID-10 em nível de dois caracteres, que qualifica "o veículo ocupado pela pessoa traumatizada ... sendo o fator mais importanteque deve ser identificado para fins de prevenção" (OMS, 1996:976), ou seja, o tipo de vítima (ou o papel desempenhado pela pessoa no momento do acidente). Dessa forma, os agrupamentos foram os seguintes: pedestres (V01-V09), ciclistas (V10-V19), motociclistas (V20-V29), ocupante de carro (V40-V49), de caminhonete (V50-V59), de caminhão (V60-V69), de ônibus (V70-V79), de veículo de tração animal ou pessoa montada em animal (V80), ocupante de trator (V84), vítima de acidente de trânsito, não especificada (V89), vítima de trauma não especificado, intenção indeterminada (Y34).

A análise da cobertura dos registros policiais sobre os acidentes foi realizada tendo como parâmetro de comparação, de um lado, os bancos de dados de vítimas atendidas em prontos-socorros, internadas ou que morreram por acidentes de transporte terrestre, já reclassificadas com a informação pós-investigação e, de outro, o banco de dados sobre acidentes registrados pela polícia (BOs). Assim, para cada vítima atendida, ou que faleceu, observou-se a presença ou ausência do respectivo Boletim de Ocorrência referente ao acidente.

A análise da validade dos registros médicos foi feita comparando as proporções de tipos de vítimas por meio das informações originais e após a investigação. Para análise das concordâncias dessas informações, em pares, utilizou-se o coeficiente Kappa (Cohen, 1960), que varia de $+1,0$ (total concordância entre os pares) passando pelo zero (ausência de concordância) e podendo assumir valores negativos. O programa de domínio público EPIDAT foi utilizado para cálculo de $\kappa$ e seus respectivos intervalos de confiança de 95\% (Xunta de Galicia/OPS, 1994).

\section{Resultados}

Das 3.643 vítimas de acidentes de transporte terrestre ocorridos em Londrina, no período estudado, observou-se que 2.917 (80,1\%) tiveram atendimento apenas em pronto-socorro, 371 (10,2\%) foram internadas, com alta posterior, e 65 (1,8\%) morreram, totalizando 3.353. As restantes 290 (7,9\%) foram levantadas em BOs, mas não foram localizadas as fichas de atendimento em pronto-socorro, de internação ou declaração de óbito, o que leva à pressuposição de que não foram atendidas por apresentarem apenas ferimentos leves, ou que buscaram atendimento em outros níveis de assistência (unidades básicas de saúde) ou outros municípios, e ainda, as que foram atendidas por pagamento direto ou planos privados de saúde (Andrade \& Mello-Jorge, 2000). Na avaliação da qualidade da informação foram excluídas, visto que não havia fichas disponíveis para verificar a informação originalmente anotada. Para a análise de cobertura dos acidentes, essas 290 vítimas foram mantidas, tendo em vista que foram "cobertas" por esses registros.

\section{Qualidade da informação}

O estudo da qualidade da informação teve como base os níveis de atendimento das vítimas: atendidas em pronto-socorro (fichas de pronto-socorro), internadas (fichas de $\mathrm{AlH}$ ) e, para as que faleceram, a informação sobre as circunstâncias do acidente registrada na DO. Cabe esclarecer que a análise foi realizada em termos de fichas de atendimento, tendo em vista que o que se espera avaliar é a qualidade dos registros em cada nível. Uma vítima que tenha recebido atendimento em pronto-socorro, sendo internada e, posteriormente, tenha falecido, deveria ter, portanto, um total de três fichas, uma respectiva a cada nível. Por conseguinte, foram analisadas 3.795 fichas (3.337 de pronto-socorro, 393 de internação e 65 declarações de óbito).

A Tabela 1 mostra a comparação das informações sobre o tipo de vítima traumatizada no acidente, registradas nas respectivas fichas com a informação pós-investigação, tendo como base informações da Polícia de Trânsito, das CATs, de notícias da imprensa escrita e de entrevistas com as próprias vítimas ou familiares. Enquanto os traumas por intenção ignorada (categoria residual das causas externas de lesões) responderam por apenas 9,2\% dos atendimentos em pronto-socorro, esse percentual chega a 38,4\% e 35,4\% das informações registradas em fichas de internação e nas declarações de óbito, respectivamente. Além disso, os 
Tabela 1

Vítimas de acidentes de transporte terrestre, segundo a informação registrada sobre o tipo de vítima em fichas de pronto-socorro, internação e declarações de óbito, e na opinião do investigador. Londrina, 1996.

\begin{tabular}{|c|c|c|c|c|c|c|c|c|c|c|c|c|}
\hline \multirow[t]{3}{*}{ Tipo de vítima* } & \multicolumn{4}{|c|}{ Pronto-socorro } & \multicolumn{4}{|c|}{ Internação } & \multicolumn{4}{|c|}{ Ó bito } \\
\hline & \multicolumn{2}{|c|}{ Original } & \multicolumn{2}{|c|}{ Pós-investigação } & \multicolumn{2}{|c|}{ Original } & \multicolumn{2}{|c|}{ Pós-investigação } & \multicolumn{2}{|c|}{ Original } & \multicolumn{2}{|c|}{ Pós-investigação } \\
\hline & $\mathrm{n}$ & $\%$ & $\mathrm{n}$ & $\%$ & $\mathrm{n}$ & $\%$ & $\mathrm{n}$ & $\%$ & $\mathrm{n}$ & $\%$ & $n$ & $\%$ \\
\hline Pedestre (V01-V09) & 358 & 10,7 & 386 & 11,6 & 57 & 14,5 & 74 & 18,8 & 12 & 18,5 & 20 & 30,8 \\
\hline Ciclista (V10-V19) & 606 & 18,2 & 723 & 21,7 & 18 & 4,6 & 57 & 14,5 & - & - & 6 & 9,2 \\
\hline Motociclista (V20-V29) & 1.076 & 32,2 & 1.450 & 43,5 & 79 & 20,1 & 183 & 46,6 & 1 & 1,5 & 27 & 41,5 \\
\hline $\begin{array}{l}\text { O cupante de carro } \\
\text { (V40-V49) }\end{array}$ & 318 & 9,5 & 548 & 16,4 & 42 & 10,7 & 50 & 12,7 & - & - & 8 & 12,3 \\
\hline $\begin{array}{l}\text { O cupante de } \\
\text { caminhonete (V50-V59) }\end{array}$ & 3 & 0,1 & 22 & 0,7 & 1 & 0,3 & 7 & 1,8 & - & - & 2 & 3,1 \\
\hline $\begin{array}{l}\text { O cupante de } \\
\text { caminhão (V60-V69) }\end{array}$ & 21 & 0,6 & 25 & 0,7 & 1 & 0,3 & 4 & 1,0 & - & - & 1 & 1,5 \\
\hline $\begin{array}{l}\text { O cupante de ônibus } \\
\text { (V70-V79) }\end{array}$ & 28 & 0,8 & 52 & 1,6 & - & - & 5 & 1,3 & - & - & - & - \\
\hline $\begin{array}{l}\text { Pessoa em animal/ } \\
\text { veículo tração animal (V80) }\end{array}$ & 15 & 0,4 & 16 & 0,5 & - & - & - & - & - & - & - & - \\
\hline O cupante de trator (V84) & 4 & 0,1 & 5 & 0,1 & 1 & 0,3 & 1 & 0,3 & - & - & 1 & 1,5 \\
\hline $\begin{array}{l}\text { Acidentes de trânsito (vítima } \\
\text { não especificada) (V89) }\end{array}$ & 600 & 18,0 & 110 & 3,3 & 43 & 10,9 & 12 & 3,0 & 29 & 44,6 & - & - \\
\hline $\begin{array}{l}\text { Trauma não especificado } \\
\text { (intenção ignorada) (Y34) }\end{array}$ & 308 & 9,2 & - & - & 151 & 38,4 & - & - & 23 & 35,4 & - & - \\
\hline Total & 3.337 & $100,0 * *$ & 3.337 & $100,0 * *$ & 393 & 100,0 & 393 & 100,0 & 65 & 100,0 & 65 & $100,0 * *$ \\
\hline
\end{tabular}

* Os códigos entre parênteses se referem aos da CID-10 (O MS, 1996).

** Arredondado para $100,0 \%$.

acidentes de trânsito sem especificação do tipo de vítima (categoria residual dos "acidentes de transporte terrestre") perfizeram quase a metade $(44,6 \%)$ das causas informadas de óbitos por esse subgrupo, com uma proporção menor nas fichas de internação (10,9\%) e de pronto-socorro (18,0\%).

Quanto ao tipo de vítima, observa-se, após investigação, maior incremento na proporção de motociclistas, que aumentaram sua contribuição no conjunto das vítimas, de 32,2\% para $43,5 \%$ (atendidos em pronto-socorro) de $20,1 \%$ para $46,6 \%$ (internados) e de $1,5 \%$ para $41,5 \%$ (óbitos).

Comparando a informação originalmente registrada sobre o tipo de vítima em cada ficha ou declaração de óbito com a informação pósinvestigação, de forma pareada, observa-se baixa concordância, principalmente com relação às registradas nas declarações de óbito, cujo coeficiente Kappa atinge apenas 0,1 (Tabela 2).

Observou-se, ainda entre as discordâncias, que nas fichas médicas de cinqüenta ciclistas e 19 motociclistas atendidos em pronto-socorro, constava na descrição do acidente, apenas a palavra "atropelamento", sendo classificados, inicialmente, como pedestres, conforme determina a CID-10 (OMS, 1996). Esse fato também foi observado entre as vítimas internadas (dez ciclistas e quatro motociclistas) e em declarações de óbito (três ciclistas).

\section{Cobertura dos registros policiais}

Do total de vítimas, menos de um terço (32,5\%) dispunha do respectivo BO policial sobre o acidente. Dependendo do papel desempenhado pela pessoa no momento do acidente, todavia, essa cobertura foi ainda menor, como pode ser observado na Tabela 3. As maiores coberturas foram observadas para ocupantes de carro $(71,6 \%)$ e de caminhonete $(55,6 \%)$ e as menores - excluindo os ocupantes de trator e de veículos de tração animal ou pessoas montadas em animais, em razão do pequeno número - entre ciclistas e pedestres.

Entre os distintos tipos de vítima, a maior cobertura foi atingida quando houve envolvimento de carro/caminhonete ou veículo de transporte pesado/ônibus. Para os pedestres, por exemplo, houve uma variação desde 2,6\% (quando atropelado por bicicleta) até 72,2\% (quando atropelado por veículo de transporte pesado ou ônibus).

No caso dos ciclistas, a cobertura variou de $12,5 \%$, quando estes colidiram com pedestre 
ou animal, até $50,0 \%$ (colisão com veículo de transporte pesado ou ônibus).

Para os motociclistas, a variação foi de 7,6\% (quedas, sem colisão) até $68,6 \%$ (colisão com veículo de transporte pesado ou ônibus).

Já entre os ocupantes de carro, que apresentaram, em média, a melhor cobertura, observou-se que nos casos de vítimas traumatizadas sem colisão a cobertura policial foi bastante baixa $(27,1 \%)$ chegando a atingir $85,6 \%$ quando a colisão se deu com outro automóvel.

A Tabela 4 apresenta a cobertura dos registros policiais (BO) em relação às vítimas sobreviventes e que morreram (em um período de até 180 dias) em conseqüência do acidente. Nota-se, com relação aos sobreviventes, uma cobertura média de $31,5 \%$, enquanto para os que faleceram a cobertura foi bem mais elevada $(86,2 \%)$. Entre os que sobreviveram, a maior cobertura foi observada para os que ficaram internados $(48,8 \%$, contra $22,5 \%$ dos que foram atendidos apenas em pronto-socorro). Para as 290 vítimas que foram listadas em BO, mas para as quais não foram localizadas fichas de atendimento médico pelo SUS ou declaração de óbito, embora não se saiba se foram apenas atendidas em pronto-socorro ou se foram internadas, pressupôs-se que sobreviveram ao acidente, considerando que a declaração de óbito é universal e, em Londrina, o Instituto Médico Legal (IML), responsável por seu fornecimento, tem abrangência regional.

\section{Discussão}

A cobertura dos registros policiais para os acidentes de transporte terrestre ocorridos em Londrina, observada no presente trabal ho, foi bastante baixa, pois para cerca de dois terços das vítimas o $\mathrm{BO}$ do acidente não havia sido preenchido. Na realidade, seria de se esperar que essa cobertura fosse inadequada para acidentes de ocupantes de trator, bicicletas e de pessoas montadas em animal ou ocupando veículo de tração animal, considerando que o primeiro é um tipo de veículo agrícola, mais propenso à ocorrência de acidentes fora da via pública (não de trânsito), o mesmo ocorrendo para vítimas montadas em animais ou em veículos de tração animal. Com relação à bicicleta, tratando-se de um veículo a pedal, sem motor, com possibilidade de grande parte dos acidentes serem decorrentes de quedas isoladas, também não seria esperada uma grande taxa de notificação à Polícia. No entanto, foram baixas, também, as proporções de vítimas pedestres $(24,8 \%)$ e motociclistas $(30,4 \%)$ que tive-
Tabela 2

Concordância entre pares de informação (originais e pós-investigação) em fichas de pronto-socorro, internação e óbito de vítimas de acidentes de transporte terrestre. Londrina, 1996.

\begin{tabular}{lcc}
\hline Fichas & Kappa & IC 95\% \\
\hline Pronto-socorro & 0,63 & $0,61-0,65$ \\
Internação & 0,33 & $0,27-0,40$ \\
Óbito & 0,10 & $0,02-0,17$ \\
\hline
\end{tabular}

Tabela 3

Distribuição de vítimas de acidentes de transporte terrestre, segundo o tipo de vítima, no momento do acidente e a cobertura de registros policiais. Londrina, 1996.

\begin{tabular}{lrrr}
\hline Tipo de vítima* & \multicolumn{2}{c}{ Cobertura } & Total \\
& n & $\%$ & \\
\hline Pedestre (V01-V09) & 102 & 24,8 & 411 \\
Ciclista (V10-V19) & 60 & 8,1 & 738 \\
Motociclista (V20-V29) & 478 & 30,4 & 1.570 \\
Ocupante de carro (V40-V49) & 487 & 71,6 & 680 \\
Ocupante de caminhonete (V50-V59) & 15 & 55,6 & 27 \\
Ocupante de caminhão (V60-V69) & 14 & 45,2 & 31 \\
Ocupante de ônibus (V70-V79) & 25 & 46,3 & 54 \\
Pessoa em animal/veículo tração animal (V80) & 2 & 11,8 & 17 \\
Ocupante de trator (V84) & - & - & 5 \\
Ignorada (V89) & - & - & 110 \\
Total & 1.183 & 32,5 & 3.643 \\
\hline
\end{tabular}

* Os códigos entre parênteses se referem aos códigos da CID-10 (O M S, 1996).

Tabela 4

Distribuição (n e \%) de vítimas de acidentes de transporte terrestre, segundo sua evolução/nível de atendimento e a cobertura de registros policiais. Londrina, 1996.

\begin{tabular}{lrrr}
\hline Evolução/nível de atendimento & \multicolumn{2}{c}{ Cobertura } & Total \\
& \multicolumn{1}{c}{$n$} & $\%$ & \\
\hline Sobreviventes & 1.127 & 31,5 & 3.578 \\
Pronto-socorro & $(656)$ & $(22,5)$ & $(2.917)$ \\
Internação & $(181)$ & $(48,8)$ & $(371)$ \\
Ignorado & $(290)$ & $(100,0)$ & $(290)$ \\
Óbito & 56 & 86,2 & 65 \\
Total & 1.183 & 32,5 & 3.643 \\
\hline
\end{tabular}


ram registrados seus acidentes em $\mathrm{BOs,} \mathrm{embo-}$ ra a maioria tenha se traumatizado em colisão com veículo a motor na via pública.

É possível que as pessoas envolvidas nesses acidentes não sintam necessidade ou não queiram convocar a Polícia de Trânsito para registro da ocorrência por razões diversas. O presente estudo, no entanto, não foi planejado para avaliar os motivos que levaram a não realização de BOs sobre o acidente.

Falhas na cobertura com relação às vítimas podem comprometer a validade de estudos que trabalhem com esses tipos de registros, principalmente no que diz respeito às pessoas que sofrem traumas mais leves e àquelas que desempenham determinados papéis no momento do acidente, como é o caso de pedestres, ciclistas e motociclistas, como demonstrado neste trabalho.

Essa realidade, contudo, parece não se limitar ao município de Londrina, pois mesmo em países desenvolvidos há relatos de sub-registro de acidentes pelos órgãos responsáveis por essas informações. Teanby (1992) verificou, por exemplo, em uma região da Grã-Bretanha, que para $16 \%$ dos pedestres falecidos não havia o correspondente registro policial. No Canadá, observou-se que $40 \%$ das crianças menores de 15 anos atendidas em setores de emergência em decorrência de acidentes, como pedestres ou ciclistas, não tinham registro do evento na polícia (Joly et al., 1991). Na Austrália, Cercarelli et al. (1996) verificaram, como no atual estudo, uma decrescente cobertura de registro policial do acidente à medida que a gravidade do trauma diminuía: $88,9 \%$ para os que morreram, $69,9 \%$ para os internados e $45,2 \%$ para os atendidos em setor de emergência com alta posterior. Nesse mesmo país, observou-se, em outro estudo, que, em média, apenas $64 \%$ das vítimas atendidas em hospitais dispunham de registro policial, sendo menor a proporção para motociclistas em acidentes envolvendo apenas a motocicleta (29\%) em comparação aos condutores dos demais veículos a motor (79\%) (Rosman \& Knuiman, 1994). Em estudo realizado na França, Aptel et al. (1999) detectaram taxas significativamente menores de notificação do acidente à polícia entre pedestres e motociclistas e entre vítimas com concentração sangüínea de álcool igual ou maior que $0,8 \mathrm{~g} / \mathrm{l}$. Por outro lado, esse mesmo trabalho encontrou maiores taxas para os acidentes ocorridos na área urbana, entre 6 e 24 horas, e entre os que permaneceram internados durante mais de seis dias.

Os resultados da presente investigação indicam que os dados de acidentes de trânsito derivados de registros policiais apresentam faIhas importantes do ponto de vista quantitativo, pois mais de dois terços das vítimas não teriam sido captadas, caso não fossem consultadas outras fontes de dados, como as declarações de óbito e as fichas hospitalares. Não obstante esse fato, esses registros fornecem valiosos detal hes a respeito de características relacionadas ao veículo, às vítimas, à via pública e às condições sob as quais se deram os acidentes, importantes para subsidiar a adoção de medidas apropriadas de prevenção. Esses registros podem ser utilizados, ainda, na avaliação de tendências, porque são os únicos que possibilitam a obtenção de dados sobre vítimas não fatais em uma série histórica relativamente longa. Cuidado especial deve ser tomado, entretanto, nas conclusões referentes a vítimas específicas, como ciclistas e pedestres, bem como para as vítimas com trauma de menor gravidade, tendo em vista sua evidente subestimação nesses boletins.

Para a análise da mortalidade por essas causas, recomenda-se trabalhar com dados da área da Saúde (o SIM ) pois, apesar de problemas relativos à qualidade da informação (Mello-jorge, 1990; Mello-Jorge et al., 1997), estes permitem uma cobertura melhor do que os registros policiais, ainda que não possibilitem discriminar o município de ocorrência do acidente, pois as informações disponíveis, referem-se apenas ao local de residência ou ocorrência do óbito.

Com relação à qualidade da informação sobre as circunstâncias do acidente, deficiências já foram salientadas por diversos pesquisadores brasileiros em relação aos dados de mortalidade (Andrade, 1995; Drumond Jr. et al., 1999; Ladeira e Guimarães, 1998; Mello-Jorge, 1990) e alguns municípios têm implantado ações com vistas a contornar tal problema, como é o caso de Londrina, através de seu Núcleo de Informações em Mortalidade (Silva \& Andrade, 1996).

No que concerne à morbidade, Lebrão et al . (1997) lamentam o fato de que, nas internações hospitalares via SUS, praticamente inexistirem dados que permitam o conhecimento de quais causas externas provocaram lesões e envenenamentos, o que foi sanado a partir de 1998, em razão de determinação específica do Ministério da Saúde.

No presente trabalho, a análise das informações sobre as circunstâncias do acidente nos três níveis (pronto-socorro, internação e óbito) revelou, paradoxalmente, uma piora da qualidade à medida que a gravidade do trauma aumentou, com um gradiente decrescente de concordância, medido pelo coeficiente Kappa, do nível de pronto-socorro até o óbito $(0,63$ a 
$0,10)$. De acordo com a proposta de classificação de Landis \& Koch (1977), a concordância, nesta investigação, seria pequena para as informações contidas nas declarações de óbito, regular para as descritas nas fichas de internação e substancial para as de pronto-socorro.

A declaração de óbito, preenchida com base em necropsia e laudos policiais, deixou de informar como acidentes de transporte terrestre cerca de $35 \%$ das mortes. No conjunto de declarações, chama ainda a atenção o fato de que apenas $20,0 \%$ definiam o tipo de vítima (18,5\% classificadas como pedestres e 1,5\% como motociclista) e em cerca de $45 \%$ desses óbitos constava apenas "acidente de trânsito", sem especificação do tipo de vítima. Caso nenhuma investigação fosse realizada, os ciclistas não seriam representados entre os óbitos e os motociclistas, que foram maioria, representariam apenas $1,5 \%$.

O percentual de identificação dos óbitos por acidentes de transporte terrestre observado neste estudo (65\%) está bastante abaixo do encontrado em uma coorte de veteranos americanos combatentes no Vietnã (97\%) (Moyer et al., 1989), e é semelhante ao observado em Belo Horizonte, Minas Gerais (68\%) (Ladeira \& Guimarães, 1998). Em São Paulo, Drumond Jr. et al. (1999) relatam uma melhor qualidade: em 1996, os atropelamentos, acidentes ferroviários e outros acidentes de trânsito foram revelados em 1.725 óbitos, perfazendo $93,4 \%$ do total de mortes por essas causas após investigação. Os resultados aqui apresentados denotam a necessidade de se investir na melhoria da informação em mortalidade por causas externas na sua origem, a partir da declaração de óbito, o que pode ser atingido por intermédio de investimentos junto ao IML e estudantes de Medicina.

Cumpre assinalar que, tanto em Londrina como em São Paulo, há programas municipais específicos de melhoria da informação oficial em mortalidade (Drumond Jr. et al., 1999; Silva \& Andrade, 1996). Dessa maneira, a situação precária da informação revelada nos resultados apresentados é, quase que totalmente, retificada pelo Núcleo de Informações em Mortalidade de Londrina (NIM) que, através de consultas ao próprio IML, imprensa escrita ou família, faz correções nas causas básicas de óbito que constarão do sistema oficial de informações em mortalidade.

Com relação às informações registradas em fichas de prontos-socorros e de internação, apesar dessas apresentarem melhor qualidade do que as de mortalidade, observa-se, ainda, uma parcela importante de traumas de intenção indeterminada (principalmente nos inter- nados) e de vítimas cujo papel no momento do acidente não é especificado. Nesses casos, assim como nos óbitos, cabe lembrar que a introdução da CID-10, em substituição à nona revisão, trouxe modificações substanciais quanto à necessidade de informação para codificação (Laurenti, 1997), sendo recente seu uso em nosso meio (desde 1996, para dados de mortalidade, e desde 1998 para as internações pelo SUS). Nos acidentes de transporte terrestre, as vítimas passaram a ser classificadas, principalmente, por seu papel no momento do acidente (pedestre, ciclista, motociclista, etc.) e, secundariamente, pelas características do acidente (colisão com ônibus, carro, caminhão, acidente sem colisão, etc.). O quarto dígito é reservado para maior especificação, como por exemplo se a vítima era condutora ou passageira, se o acidente ocorreu na via pública, entre outros. Esses detalhes contribuem, sem dúvida, para a indicação de situações que favorecem a ocorrência dos acidentes de transporte. Dessa forma, acredita-se que seja necessária uma maior divulgação da Classificação de Doenças em vigor, visando estimular diagnósticos que possibilitem uma classificação acurada, coerente com o propósito da informação sobre causas externas, qual seja, o de subsidiar as ações que tenham como meta a redução desse fenômeno.

Outra característica mostrada neste estudo é a de que o termo "atropelamento", sem qualquer outra especificação, tem sido utilizado para outras categorias de vítimas, além de pedestres. De acordo com a CID-10 (OMS, 1996), em seu capítulo de “instruções para a classificação e a codificação de acidentes de transporte", se a condição da vítima não estiver especificada, mas constar que foi atropelada, esta deve ser classificada como pedestre (categorias V01-V09). Notou-se neste estudo, no entanto, que algumas das vítimas que ocupavam bicicleta ou motocicleta receberam essa classificação (atropelamento) sem qual quer menção ao veículo ocupado, o que faria, caso não houvesse investigação complementar, que fossem classificadas como pedestres, inflacionando essa categoria. No caso de óbitos, o aumento proporcional seria ainda maior, dado o menor número de eventos. Esse procedimento, caso assuma maiores proporções, pode comprometer a análise de dados que se baseiem apenas na descrição do acidente realizada pelo profissional de saúde, com subestimação do número de ciclistas e motociclistas traumatizados em acidentes.

A situação apresentada com relação à qualidade da informação revela, por um lado, pouco conhecimento e/ ou motivação dos profissionais de saúde que atendem essas vítimas 
quanto às necessidades de detal hamento, nos casos de acidente de transporte, do papel desempenhado pela vítima e das circunstâncias do acidente, para uma classificação detalhada e internacionalmente recomendada, como é o caso da CID-10. Por outro, demonstra, como seria de se esperar, maior preocupação desses profissionais com relação à descrição das lesões, o que é necessário para a adequada e oportuna instalação de medidas de recuperação das funções das vítimas. Todavia, como já referido, a causa externa que provocou a lesão tem importância fundamental em nível da saú-

\section{Referências}

ANDRADE, S. M., 1995. Aspectos da situação das estatísticas oficiais da mortalidade por causas externas no Município de Londrina - Paraná. Semina, 16:300-308.

ANDRADE, S. M. \& MELLO-JORGE, M. H. P., 2000. Características das vítimas por acidentes de transporte terrestre em município da Região Sul do Brasil. Revista de SaúdePública, 34:149-156.

APTEL, I.; SALMI, L. R.; MASSON, F.; BOURDÉ, A.; HENRION, G. \& ERNY, P., 1999. Road accidents statistics: Discrepancies between police and hospital data in a French island. Accident Analysis and Prevention, 31:101-108.

CDC (Centers for Disease Control and Prevention)/ WHO (World Health Organization), 1996. Epi Info 6, Versi on 6.04: A Word Processing, Database, and Statistics Program for Public Health. Atlanta: CDC/Geneva: WHO.

CERCARELLI, L. R.; ROSMAN, D. L. \& RYAN, G. A., 1996. Comparison of accident and emergency with police road injury data. Journal of Trauma, 40:805-809.

COHEN, J., 1960. A coefficient of agreement for nominal scales. Educational and Psychological Measurement, 10:37-46.

DRUMOND Jr., M.; LIRA, M. T. A.; FREITAS, M.; NITRINI, T. M. V. \& SHIBAO, K., 1999. Avaliação da qualidade das informações de mortalidade por acidentes não especificados e eventos com intenção indeterminada. Revista deSaúdePública, 33:273-280.

IBGE (Fundação Instituto Brasileiro de Geografia e Estatística), s.d. Contagem da População 1996: Dados Distritais - População por Situação e Sexo. Rio de Janeiro: IBGE.

JOLY, M. F.; FOGGIN, P. M. \& PLESS, B., 1991. Geographical and socio-ecological variations of traffic accidents among children. Social Science and Medicine, 33:765-769.

LADEIRA, R. M. \& GUIMARÃES, M. D. C., 1998. Análise da concordância da codificação de causa básica de óbito por acidentes de trânsito. Revista deSaúdePública, 32:133-137.

LANDIS, J. R. \& KOCH, G. G., 1977. The measurement of observer agreement for categorical data. Biometrics, 33:159-174.

LAPIDUS, G.; BRADDOCK, M.; SCHWARTZ, R. BANCO, L. \& LENWORTH, J., 1994. Accuracy of fatal de coletiva, pois define as características dos acidentes ou violências responsáveis por essa demanda, sendo importante para indicar que medidas preventivas devem ser adotadas, prioritariamente, em benefício da coletividade. Daí, portanto, a necessidade de se estabelecer mecanismos que propiciem, a médio prazo, a possibilidade de se conhecer, com adequada cobertura e qualidade, e de forma rotineira, a situação também no que se refere às vítimas não fatais de acidentes de transporte terrestre em Londrina, a exemplo do que já vem ocorrendo em termos de mortalidade.

motorcycle-injury reporting on death certificates. Accident Analysis and Prevention, 26:535-542.

LAURENTI, R., 1997. Acidentes e violências/lesões e envenenamentos e a 10a revisão da Classificação Internacional de Doenças. Revista de Saúde Pública, 31(Sup. 4):55-58.

LEBRÃO, M. L.; MELLO-JORGE, M. H. P. \& LAURENTI, R., 1997. Morbidade hospitalar por lesões e envenenamentos. Revista de Saúde Pública, 31 (Sup.):26-37.

MELLO-JORGE, M. H. P., 1980. Mortalidade por causas violentas no Município de São Paulo, Brasil. II. Mortes acidentais. Revista de Saúde Pública, 14:475-508.

MELLO-JORGE, M. H. P., 1990. Situação atual das estatísticas oficiais relativas à mortalidade por causas externas. Revista de Saúde Pública, 24:217-223.

MELLO-JORGE, M. H. P.; GAWRYSZEWSKI, V. P. \& LATORRE, M. R. D. O., 1997. Acidentes e violência no Brasil. I. Análise dos dados de mortalidade. Revista de SaúdePública, 31(Sup.):5-25.

MINAYO, M. C. S. \& SOUZA, E. R., 1993. Violência para todos. Cadernos de SaúdePública, 9:65-78.

MOYER, L. A.; BOYLE, C. A. \& POLLOCK, D. A., 1989. Validity of death certificates for injury-related causes of death. American Journal of Epidemiology, 130:1024-1032.

OMS (Organização Mundial da Saúde), 1996. CID-10 - Manual de Classificação Estatística Internacional de Doenças e Problemas Relacionados à Saúde. Décima Revisão. São Paulo: Centro Colaborador da OMS para Classificação de Doenças em Português.

ROSMAN, D. L. \& KNUIMAN, M. W., 1994. A comparison of hospital and police injury road data. Accident Analysis and Prevention, 26:215-222.

SILVA, S. F. \& ANDRADE, S. M., 1996. Acidentes de trânsito: Problema prioritário de saúde? In: A Construção do SUS a partir do Município (S. F. Silva, org.), pp. 95-99, São Paulo: Editora Hucitec.

TEANBY, D., 1992. Underreporting of pedestrian road accidents. BMJ, 304:422.

XUNTA DE GALICIA/OPS (Organización Panamericana de la Salud), 1994. Epidat: Análisis Epidemiológi co de Datos Tabulados, Versión 1.0. Santiago de Compostela: Servicio Gallego de Salud/Washington, DC: OPS. 